\title{
Human T cell repertoire: what happens in thymus does not stay in thymus
}

\author{
Antonio La Cava
}

Department of Medicine, UCLA, Los Angeles, California, USA.

\begin{abstract}
The T cell receptor (TCR) repertoire is diverse, thus allowing recognition of a wide range of pathogens by $T$ cells. In humans, the study of the formation of TCR repertoires is problematic because of the difficulty in performing investigations in vivo. In this issue of the $J C I$, Khosravi-Maharlooei and colleagues describe a new humanized mouse model that allows direct investigations on this topic. Using high-throughput and single-cell TCRcomplementarity-determining region $3 \beta$ (TCR-CDR3 $\beta$ ) sequencing, the authors were able to demonstrate that human thymic selection is a major driver of TCR sequence sharing, also implicating a preferential selection of shared cross-reactive CDR3 $\beta$ s during repertoire formation.
\end{abstract}

\section{Shaping the T cell repertoire}

The generation of a broad variety of $\mathrm{T}$ cell receptors (TCRs) during $\mathrm{T}$ cell development in the thymus creates $\mathrm{T}$ cell repertoires capable of recognizing large numbers of antigens through multitudes of different TCRs. By recombination, random insertion, deletion, and substitution, the rather small set of genes that encode the TCR can create a great diversity, with the complementaritydetermining region 3 (CDR3), which serves as the primary site of antigen contact, accounting for most of the variation within an individual's $\mathrm{T}$ cell repertoire. Moreover, this CDR3 diversity is primarily affected at the level of the $\beta$-chain (CDR3ß) (1).

The host's T cell pool is shaped through a process by which TCRexpressing thymocytes undergo positive and negative selection of self-peptideMHC complexes in the thymus, thereby generating naive $\mathrm{T}$ cells that are both self-tolerant and self-MHC restricted (2). However, a consequence of self-MHC restriction is that all peripheral $\mathrm{T}$ cells are essentially self-reactive, explaining why the peripheral $\mathrm{T}$ cell repertoire contains an elevated frequency of autoreactive $\mathrm{T}$ cells, even in healthy individuals (3). Incidentally, this aspect also creates grounds for the possible development of autoimmune responses that therefore need to be kept under constant control throughout life by multiple, often complementary, mechanisms (4).

Of note, although TCR gene rearrangement is believed to occur randomly, some clonotypes are more commonly produced than others (5), leading to unequal frequencies of naive $\mathrm{T}$ cell clonotypes and so-called "public" clonotypes that are shared among individuals (6). This difference in frequency distribution has been the subject of several investigations that have resulted in varied explanations but no consensus. As a result, although a dissection of the events that lead to the formation and diversification of the human TCR repertoire remains critical to understanding the mechanisms of $\mathrm{T}$ cell development and diversity, ethical constraints and lack of suitable models have complicated the study of the formation of human TCR repertoires in vivo.

Delated Article: p. 2446

Conflict of interest: The author has declared that no conflict of interest exists.

New insights from a humanized mouse model

In this issue, Khosravi-Maharlooei and coworkers monitored TCR formation and outcomes in humanized mice that had been engrafted with human fetal thymus and human hematopoietic stem cells (HSCs) (7). This strategy allowed a recapitulation of critical mechanisms of in vivo formation of the human TCR repertoire under syngeneic and allogeneic conditions, mimicking the development of human thymocytes under conditions that are as close as possible to physiological settings. Through high-throughput and single-cell TCR $\beta$-CDR3 sequencing analyses of human thymocytes in human thymi (and in the periphery) of humanized mice, the authors were able to accredit human thymic selection as a major driver of TCR sequence sharing and implicate a preferential selection of shared cross-reactive CDR3 $\beta$ s during repertoire formation. As such, this study by Khosravi-Maharlooei and colleagues delineates the impact of human thymic selection on shared TCR sequences and their presence among mature T cells.

Khosravi-Maharlooei and coworkers analyzed the formation of the human TCR repertoire under different conditions and compared data from three different groups of humanized mice. The first group of animals received the same fetal liver HSCs and autologous fetal thymus, resulting in the same genetic background and TCR selection in the same thymus. The second group of mice also had the same genetic background and same fetal liver HSCs, together with either an autologous fetal thymus or an allogeneic fetal thymus. This group of mice allowed evaluation of thymic selection in a different thymus, with the $\mathrm{T}$ cells in the grafted thymus derived from HSCs given intravenously. Finally, the third group of mice consisted of thymectomized animals transplanted with the same fetal liver HSCs and autologous fetal thymus. Cell sequencing was done for both 
thymocytes and peripheral $\mathrm{CD} 4^{+}$and $\mathrm{CD} 8^{+}$ $\mathrm{T}$ cells, and combined approaches ensured the elimination of possibly confounding preexisting thymocytes and their progeny from transplanted thymic tissue (8).

With this model, comparisons between replicate mice allowed Khosravi-Maharlooei et al. to discern whether exposure to the same antigens for positive and negative selection in the thymus from the same human donor leads to the formation of similar TCR repertoires in different individuals. The model also allowed examination of the effects of positive and negative selection on the human TCR repertoire and the analysis of V-J pairings, as discussed below.

The first notable finding was that replicate humanized mice generated divergent repertoires. Low clonality among different human thymi underscored the selection of highly diverse repertoires, and the divergence of TCR $\beta$ repertoires in thymocytes generated in identical thymi from the same HSCs reflected the stochastic nature of TCR repertoire formation. These analyses also showed that thymic selection narrowed the $\mathrm{T}$ cell repertoire and increased CDR3 $\beta$ sharing, with augmented clonality and a progressive decrease in diversity in conjunction with $\mathrm{T}$ cell maturation. Indeed, despite the stochastic nature of the process, thymic selection favored the selection of shared sequences between individuals (with a lower divergence at the amino acid level than at the nucleotide level). This result indicated that while stochastic formation of the repertoire results in differences between replicates, there was nonetheless a preferential selection of public sequences by disparate HLA alleles, which is in line with studies that have shown a similar overlap between TCR repertoires of monozygotic twins and TCR repertoires of unrelated individuals (9). Further analyses of the frequency of the shared CDR3 $\beta$ s at the double-positive thymocyte stage compared with single-positive and peripheral $\mathrm{CD}^{+}$and $\mathrm{CD}^{+} \mathrm{T}$ cells indicated that both positive thymocyte selection and negative thymocyte selection were responsible for the increased CDR3 $\beta$ overlap between individual human $\mathrm{T}$ cell repertoires.

Structurally, the shared CDR3 $\beta$ s were shorter in length because of fewer numbers of inserted nucleotides at the V-D and D-J junctions. Moreover, CDR3 $\beta$ shortening continued further during the transition from thymic double-positive to single-positive to peripheral $\mathrm{CD}^{+}$and $\mathrm{CD}^{+} \mathrm{T}$ cells, consistent with past studies $(10,11)$. As CDR3 $\beta$ shortening within the most abundant, but not the less abundant, sequences accompanied the progression of thymic selection $\left(\mathrm{CD} 9^{+}\right.$doublepositive to single-positive transition), Khosravi-Maharlooei et al. suggested that shorter, more common CDR3 $\beta$ s could avoid negative selection and facilitate positive selection due to a lower affinity for cross-reactivity with diverse MHC peptides. Notably, shared CDR3 $\beta$ s (in singlepositive $\mathrm{CD}^{+} \mathrm{T}$ cells) had different $\mathrm{V}$ gene, but not J gene, usage, with approximately $20 \%$ to $25 \%$ of shared CDR3 $\beta$ s using the same V-J pair. The same TCR $\beta$ chain almost always paired with different $\alpha$ chains, and thymic selection did not appear to influence the overall $\mathrm{V}$ and J gene usage.

Shared CDR3 $\beta$ s also displayed an increased likelihood of cross-reactivity as compared with unshared CDR3 $\beta$ s in mixed lymphocyte reactions (HLA-mismatched); therefore, Khosravi-Maharlooei et al. wondered whether those shared sequences might be enriched for autoreactivity. Taking advantage of data from the network for Pancreatic Organ donors with Diabetes (nPOD) program (12), the authors tested for autoreactivity by identifying shared sequences in type 1 diabetes-associated (T1D-associated) CDR3 $\beta$ and CDR3 $\beta$ s amino acid sequences reactive to islet self-antigens. The finding of higher T1D autoreactivity for the shared versus nonshared CDR3 $\beta$ sequences supports the possibility that autoreactive TCRs shared by different individuals are indeed preferentially selected. Also, the investigation of the amino acid usage at each position of the CDR3 $\beta$ suggests that selected shared sequences could have weaker interactions with self-peptides than unshared sequences. In particular, the increased hydrophobic amino acid usage at positions 6 and 7, which influence self-peptide-MHC complex reactivity during positive and negative selection (13), during human thymocyte positive selection was lost in the transition from single-positive thymocytes to peripheral $\mathrm{T}$ cells, i.e., after negative selection. Considering that positive selection favored shorter shared sequences with reduced hydropho- bicity at positions 6 and 7 of the CDR3 $\beta$ s, the occurrence of weaker interactions with self-peptides (due to low affinity for self-peptide-MHC) for the shared versus unshared sequences could promote these sequences by facilitating their escape from negative selection (14).

\section{Conclusions}

In all, the study by Khosravi-Maharlooei and colleagues advances the current understanding of basic mechanisms of human TCR repertoire formation and T cell development. Human thymi formed varied TCR repertoires that diverged in the presence of the same genetic background. Thymic selection narrowed those TCR repertoires, promoting an overlap among human TCR $\beta$ sequences in which shared sequences had shorter CDR3 $\beta$ lengths and were cross-reactive and autoreactive. While previous work has suggested a role for recombination bias or convergent recombination in the generation of public sequences (15), the work of KhosraviMaharlooei et al. delineates a role of human thymic selection in enriching public TCR $\beta$ sequences, in which distinctive characteristics of the CDR3 $\beta$ s could favor positive selection and escape negative selection.

Address correspondence to: Antonio La Cava, 1000 Veteran Avenue 32-59, Department of Medicine, UCLA, Los Angeles, California 90095, USA. Phone: 310.267.4975; Email: alacava@mednet. ucla.edu.

1. Kedzierska K, La Gruta NL, Stambas J, Turner SJ, Doherty PC. Tracking phenotypically and functionally distinct $\mathrm{T}$ cell subsets via $\mathrm{T}$ cell repertoire diversity. Mol Immunol. 2008;45(3):607-618.

2. Viret C, Janeway CA Jr. MHC and T cell development. Rev Immunogenet. 1999;1(1):91-104.

3. Mycko MP, et al. Cross-reactive TCR responses to self antigens presented by different MHC class II molecules. J Immunol. 2004;173(3):1689-1698.

4. La Cava A. Putting together the autoimmunity puzzle. JClin Invest. 2015;125(6):2184-2186.

5. Venturi V, Price DA, Douek DC, Davenport MP. The molecular basis for public T-cell responses? Nat Rev Immunol. 2008;8(3):231-238.

6. Madi A, et al. T-cell receptor repertoires share a restricted set of public and abundant CDR3 sequences that are associated with self-related immunity. Genome Res. 2014;24(10):1603-1612.

7. Khosravi-Maharlooei M, et al. Cross-reactive public TCR sequences undergo positive selection in the human thymic repertoire. JClin 
Invest. 2019;129(6):2446-2462.

8. Kalscheuer $\mathrm{H}$, et al. A model for personalized in vivo analysis of human immune responsiveness. Sci Transl Med. 2012;4(125):125ra30.

9. Zvyagin IV, et al. Distinctive properties of identical twins' TCR repertoires revealed by high-throughput sequencing. Proc Natl Acad Sci US A. 2014;111(16):5980-5985.

10. Nishio J, Suzuki M, Nanki T, Miyasaka N, Kohsaka H. Development of TCRB CDR3 length reper- toire of human T lymphocytes. Int Immunol. 2004;16(3):423-431.

11. Matsutani T, et al. Comparison of CDR3 length among thymocyte subpopulations: impacts of MHC and BV segment on the CDR3 shortening. Mol Immunol. 2007;44(9):2378-2387.

12. Eugster A, et al. High diversity in the TCR repertoire of GAD65 autoantigen-specific human CD4 ${ }^{+}$T cells. J Immunol. 2015;194(6):2531-2538

13. Stadinski BD, et al. Hydrophobic CDR3 residues promote the development of self-reactive T cells. Nat Immunol. 2016;17(8):946-955.

14. Greiff V, et al. Learning the high-dimensional immunogenomic features that predict public and private antibody repertoires. J Immunol. 2017;199(8):2985-2997.

15. Venturi V, et al. Sharing of T cell receptors in antigen-specific responses is driven by convergent recombination. Proc Natl Acad Sci US A. 2006;103(49):18691-18696. 